\title{
Guías de práctica clínica: evolución, metodología de elaboración y definiciones actuales
}

\section{Clinical practice guidelines: evolution, methodology of elaboration and current definitions}

Correspondencia

Fradis Eriberto Gil Olivares fradisg|@gmail.com

Recibido: 26/10/2017

Arbitrado por pares

Aprobado: 15/11/2017

Citar como: Alva Diaz C, GarcíaMostajo JA, Gil-Olivares F, Timana $R$, Pimentel P, Canelo-Aybar C. Guías de práctica clínica: evolución metodología de elaboración y definiciones actuales. Acta Med Peru. 2017:34(4):317-22

\author{
Carlos Alva Diaz ${ }^{1,2}$, Jorge A. García-Mostajo ${ }^{1,3}$, Fradis Gil-Olivares ${ }^{1,4}$, Raúl \\ Timana ${ }^{5}$, Patricia Pimentel ${ }^{5}$, Carlos Canelo-Aybar ${ }^{6}$ \\ 1 Red de Eficacia Clínica y Sanitaria - REDECS. Lima, Perú. \\ 2 Servicio de Neurología, Departamento de Medicina, Hospital Nacional Daniel Alcides Carrión. Callao, Perú. \\ 3 Hospital Guillermo Kaelin de la Fuente. Lima, Perú. \\ 4 Unidad de Gestión de la Calidad, Hospital de Emergencias Villa El Salvador. Lima, Perú. \\ 5 Instituto de Evaluación de Tecnologías en Salud e Investigación, Seguro Social de Salud. Lima, Perú. \\ 6 Centro Cochrane Iberoamericano, Instituto de Investigación Biomédica Sant Pau. Barcelona, España.
}

\section{RESUMEN}

Las guías de práctica clínica son una de las principales herramientas utilizadas por los sistemas de salud modernos y por los profesionales de la salud que practican una medicina basada en evidencias. Son instrumentos usados en beneficio del paciente y con un impacto positivo para los sistemas de salud. Su desarrollo ha ido evolucionando hasta convertirse en instrumentos confiables y de uso generalizado, siendo varios los conceptos que se han incorporado en la formulación de recomendaciones en salud: eficacia, seguridad, costo-efectividad, equidad y enfoque centralizado en la persona. Además, el desarrollo de metodologías propuestas para su elaboración y evaluación de calidad: AGREE, ADAPTE, GRADE entre otras. En el presente ensayo se hace una revisión de los aspectos relacionados a su evolución, metodología de elaboración y las definiciones actuales para su uso como herramientas en la práctica de la medicina basada en evidencias.

Palabras clave: Guía de práctica clínica; Medicina basada en la evidencia; Sistemas de salud (fuente: DeCS BIREME).

\section{ABSTRACT}

Clinical practice guidelines are one of the main tools used by modern health systems and health professionals who practice evidence-based medicine. They are instruments used to benefit the patient and have a positive impact on health systems. Its development has evolved into reliable and widely used tools, and several concepts have been included in health recommendations: effectiveness, safety, cost-effectiveness, equity and a person-centered approach. Also, development of methodologies proposed for its elaboration and quality evaluation: AGREE, ADAPTE, GRADE among others. This paper reviews the aspects of the evolution of clinical practice guideline, methodology of elaboration and the current definition for its use as tools for the practice of evidence-based medicine.

Keywords: Practice guideline; Evidence-based medicine; Health systems (source: MeSH NLM). 


\section{INTRODUCCIÓN}

La variabilidad de los procesos de atención y en la realización de los procedimientos tanto entre médicos especialistas como entre diferentes regiones geográficas es uno de los problemas que afrontan los sistemas de salud. Pacientes con los mismos problemas clínicos reciben diferentes tratamientos dependiendo también de su médico o ubicación geográfica, todo esto con impacto negativo para la salud los pacientes y para la adecuada gestión de las instituciones sanitarias ${ }^{[1,2]}$.

El uso de guías de práctica clínica (GPC) fue una estrategia que nació para hacer frente a este problema y garantizar que con su uso los pacientes puedan recibir un diagnóstico y/o manejo estándar independientemente del médico tratante o de dónde se encuentren ${ }^{[3]}$; sin embargo, se encontró que las GPC elaboradas por diferentes instituciones sobre un mismo tema no siempre son concordantes en sus recomendaciones incluso cuando se utilizan las mismas evidencias ${ }^{[4]}$.

Aunque estas discordancias muchas veces se explican por razones metodológicas en la elaboración de la GPC o interpretación particular de las evidencias en las que se basan, también podrían explicarse por diferencias en las prevalencias de las enfermedades, en las evidencias utilizadas, en la evaluación de calidad de las mismas, en la elección en los desenlaces clínicos de interés que guían las recomendaciones, preferencias y conflictos de interés de los elaboradores ${ }^{[5]}$. Además, se ha estimado que hasta un $94 \%$ de la variación en el nivel de calidad de las GPC podrían explicarse por la calidad de las evidencias utilizadas para su desarrollo ${ }^{[6]}$. Por lo tanto, a pesar que las GPC nacen para hacer frente a la variabilidad de procesos de atención, estas a su vez empiezan a desarrollarse con variabilidad y discordancia entre sí.

Para resolver este problema se propone que las GPC armonicen su proceso de elaboración mediante el uso de metodologías estandarizadas, reduzcan al mínimo posible los sesgos financieros o intelectuales de los elaboradores mediante la declaración completa de los conflictos de interés, acuerden previamente los desenlaces clínicos críticos e importantes para evaluar la eficacia de las intervenciones diagnósticos y terapéuticas y generen propuestas de nuevas investigaciones que permitan resolver las áreas de incertidumbre ${ }^{[5]}$.

\section{LOS PROBLEMAS DE CALIDAD DE LAS GPC: UNA DECISIÓN SUPRANACIONAL}

Las GPC no nacieron basadas en evidencia, sino que inicialmente fueron elaboradas por opinión de expertos y luego por consenso de expertos con una mejora progresiva en la calidad de su desarrollo a través del tiempo ${ }^{[7]}$. Estos instrumentos fueron mejorando en su metodología (criterios de calidad, grados de recomendación, entre otros) ${ }^{[8,9]}$. (Figura 1 )

La Organización Mundial de la Salud (OMS) en el año 2007 identificó que sus guías se basaban principalmente en recomendaciones de expertos y rara vez utilizaban métodos sistemáticos basados en

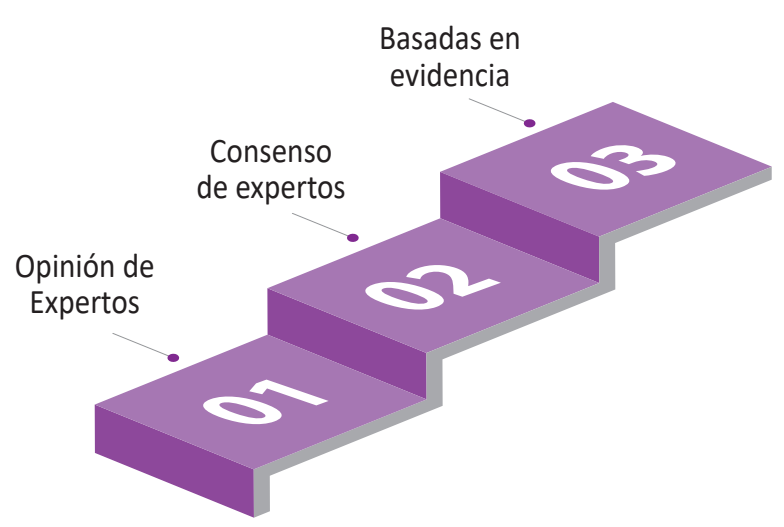

Figura 1. Evolución en el desarrollo de las guías de práctica clínica.

evidencias científicas ${ }^{[10]}$, por lo que impulsaron la creación de un comité revisor de guías (GRC, del inglés 'Guidelines Review Committee') para asegurar la calidad de las mismas mediante metodologías sistemáticas y basadas en evidencia con adopción de la metodología GRADE ${ }^{[11]}$. Esto motivo algunas mejoras en sus GPCs, los mayores cambios se registraron en los dominios "Rigor de desarrollo" (de 30,7\% a 68,3\%) e "Independencia editorial" (de $20,9 \%$ a $73,6 \%$ ), llevando a que los métodos de elaboración de guías de la OMS se tornen más sistemáticos y transparentes desde el inicio de la gestión del GRC ${ }^{[12]}$.

\section{GUIAAS DE PRÁCTICA CLÍNICA CONFIABLE}

\section{¿Qué es una GPC confiable?}

Las GPC son definidas por el Instituto de Medicina (IOM, del inglés Institute of Medicine, de Estados Unidos de América) como "declaraciones que incluyen recomendaciones destinadas a optimizar la atención al paciente que se elaboraron mediante la revisión sistemática de las evidencias existentes y la evaluación de riesgos y beneficios de las diferentes opciones de cuidados". Además, para que una GPC sea confiable, la IOM recomienda que estas deben considerar seis criterios importantes ${ }^{[13]}$. (Figura 2).

\section{GPC confiable o basada en evidencias}

El desarrollo de una GPC basadas en evidencia (GPC-BE) se inicia por la priorización sistemática y explícita de temas o condiciones clínicas ${ }^{[14,15]}$. Para la elaboración de GPC-BE se recomienda que las preguntas clínicas, que deberán ser sistematizadas mediante recomendaciones, se estructuren mediante el formato PICO (por sus siglas en inglés, Patient, intervention, comparison and outcome) o el PIPOH (por sus siglas en inglés, Population, intervention, professionals, outcome and health care) de la colaboración ADAPTE para el desarrollo de GPC adaptadas ${ }^{[16,17]}$. Luego, se identifican y seleccionan las evidencias adecuadas, se evalúa su calidad y finalmente se formulan las recomendaciones. La calidad de la evidencia debiera estar íntimamente relacionada con la fuerza de las recomendaciones, siendo GRADE uno de los métodos más ampliamente difundido y reconocido como completo ${ }^{[18-20]}$. 


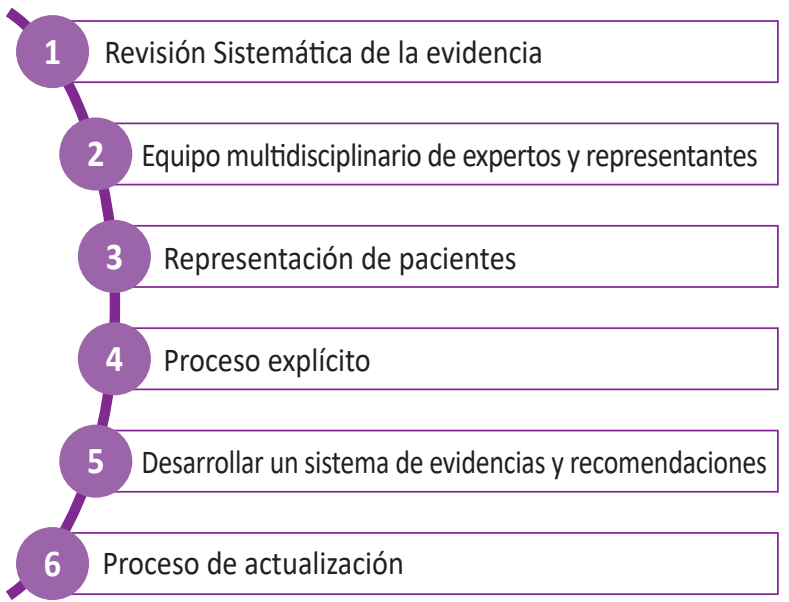

Figura 2. Guías de práctica clínica confiable (IOM).

Finalmente, para la adaptación o adopción de una GPC$B E$ es necesario valorar su calidad global y para ello se recomienda utilizar el AGREE II que es un instrumento validado internacionalmente y traducido a más de 33 idiomas para evaluar el rigor metodológico de las mismas ${ }^{[21-23]}$ (Figura 3); además, de utilizar un proceso explícito de adaptación/adopción o contextualización de una GPC-BE para lo cual se han propuesto diferentes estrategias, se han identificado 8, entre las que destacan ADAPTE, su versión corta RAPADAPTE y la novedosa GRADE-ADOLOPTMENT ${ }^{[23-25]}$. (Tabla 1)

\section{BALANCE EN EL DESARROLLO Y USO DE LAS GPC}

\section{Beneficios}

Se reconoce beneficios de las GPC-BE en primer lugar para los pacientes al mejorar la calidad del cuidado que reciben y sus resultados en salud ${ }^{[23,25]}$. Las guías al recomendar intervenciones de beneficio probado y desalentar el uso de aquellas que son ineficaces han demostrado reducir la mortalidad ${ }^{[26,27]}$, el riesgo de infecciones ${ }^{[28,29]}$ y mejorar la calidad de vida de los pacientes ${ }^{[3]}$. También se han reconocido beneficios en términos de un mayor acceso a la información mediante la difusión de versiones para los pacientes de las GPC lo que contribuye a tomar decisiones compartidas con su médico tratante en relación a las diferentes opciones de tratamiento ${ }^{[3]}$.

Para los médicos, las GPC-BE pueden mejorar la calidad de sus decisiones clínicas, aclaran qué intervenciones son de beneficio probado, reportan la calidad de los datos que la soportan e identifican a aquellas ineficaces. Por lo tanto, ofrecen recomendaciones explícitas para los médicos que dudan de cómo proceder, revierten creencias de prácticas anticuadas, mejoran la consistencia de la atención y ofrecen recomendaciones autorizadas que tranquilizan a los profesionales sobre la idoneidad de sus decisiones ${ }^{[23]}$. Además, las GPC-BE proveen herramientas para mejorar la calidad de atención como la lista de recomendaciones, rutas de cuidados críticos, algoritmos, criterios de auditoría entre otros.

Las GPC-BE son un punto de referencia para las auditorías de las prácticas clínicas y hospitalarias: las pruebas de ayuda diagnóstica, los tratamientos y los objetivos de tratamiento recomendados en las guías proporcionan medidas de proceso (criterios de revisión) para calificar el cumplimiento de las mejores prácticas de cuidado. La evaluación crítica de la evidencia, que se utilizan en el desarrollo de las GPC-BE, identifican defectos de diseño en estudios existentes y reconocen las brechas de evidencia que pueden reorientar el trabajo de los investigadores médicos y alentar a las agencias de financiamiento a apoyar investigaciones que cumplan con esta agenda basada en la efectividad ${ }^{[3]}$.

Para los sistemas de salud, tanto de proveedores públicos como privados, se ha descrito que las GPC-BE pueden ser útiles para mejorar la eficiencia en el uso de recursos mediante la estandarización de la atención con terapias de beneficio probado y costo-efectivas ${ }^{[30]}$, al reducir las tasas de admisión ${ }^{[23]}$, la duración de la estancia hospitalaria ${ }^{[23,29,31,32]}$ y los costos hospitalarios ${ }^{[23,33,34]}$. Además, la adherencia a GPC-BE mejora la imagen pública de las instituciones al garantizar un compromiso con la excelencia y la calidad ${ }^{[3]}$.

\section{Limitaciones}

A pesar de los beneficios comprobados en el uso de GPC, estas también pueden presentar limitaciones y riesgos. Una de las más importantes es que sus recomendaciones estén equivocadas 0 que no se ajusten a las necesidades del paciente individual y fomenten el uso de intervenciones ineficaces, perjudiciales o derrochadoras. Los factores son varios, siendo un ejemplo la carencia o limitaciones de las evidencias para dar respuesta a un problema de salud. Estas limitaciones pueden ser por problemas en el diseño de los estudios y riesgo de sesgos, inconsistencia de

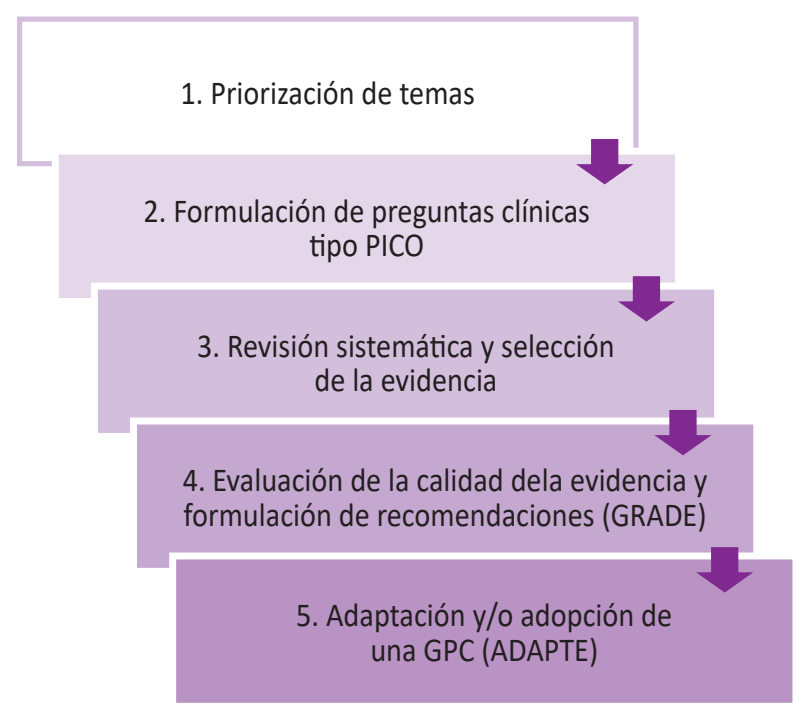

Figura 3. Desarrollo de una GPC-BE. 
Tabla 1. Comparación de métodos de desarrollo de guías de práctica clínica (GPC).

\begin{tabular}{|c|c|c|c|}
\hline Criterio & $\begin{array}{c}\text { GPC basada en opinión de } \\
\text { expertos }\end{array}$ & GPC-BE de novo & GPC-BE adaptada \\
\hline Enfoque & Narrativo & $\begin{array}{l}\text { Metodológico, basada en } \\
\text { evidencias científicas }\end{array}$ & $\begin{array}{l}\text { Metodológico, basada en } \\
\text { evidencias científicas }\end{array}$ \\
\hline Documento base & $\begin{array}{l}\text { No siempre declarado. } \\
\text { Evidencias sin valoración } \\
\text { explícita }\end{array}$ & $\begin{array}{l}\text { Evidencias primarias valoradas } \\
\text { según criterios de calidad }\end{array}$ & $\begin{array}{l}\text { GPC-BE existente valoradas según } \\
\text { criterio de calidad metodológica } \\
\text { (AGREE II) }\end{array}$ \\
\hline Grupo elaborador de la guía & $\begin{array}{l}\text { Variable, usualmente } \\
\text { sólo expertos en el tema } \\
\text { específico }\end{array}$ & $\begin{array}{l}\text { Multidisciplinario con expertos y } \\
\text { metodólogos }\end{array}$ & $\begin{array}{l}\text { Multidisciplinario con expertos y } \\
\text { metodólogos }\end{array}$ \\
\hline $\begin{array}{l}\text { Declaración de conflicto de } \\
\text { intereses }\end{array}$ & Opcional & Obligatorio & Obligatorio \\
\hline $\begin{array}{l}\text { Formulación de preguntas } \\
\text { clínicas }\end{array}$ & Sin formato establecido & Preguntas PICO & Según documento base. \\
\hline Metodología de desarrollo & No explicitó ni replicable. & $\begin{array}{l}\text { Con enfoque de MBE: revisiones } \\
\text { sistemáticas/evaluación de } \\
\text { calidad de la evidencia/sistema } \\
\text { para formular recomendaciones }\end{array}$ & $\begin{array}{l}\text { Con enfoque de MBE: revisiones } \\
\text { sistemáticas/evaluación de calidad } \\
\text { del documento base y de la nueva } \\
\text { evidencia/sistema para formular } \\
\text { recomendaciones }\end{array}$ \\
\hline $\begin{array}{l}\text { Base de datos para } \\
\text { búsqueda }\end{array}$ & $\begin{array}{l}\text { No explicitó o no } \\
\text { replicable. }\end{array}$ & $\begin{array}{l}\text { Exhaustivo: Medline, Cochrane, } \\
\text { Embase, etc. }\end{array}$ & $\begin{array}{l}\text { Exhaustivo: Medline, Cochrane, } \\
\text { Embase, etc. }\end{array}$ \\
\hline $\begin{array}{l}\text { Sistema para elaboración de } \\
\text { recomendaciones }\end{array}$ & $\begin{array}{l}\text { No explicitó o no } \\
\text { replicable. }\end{array}$ & $\begin{array}{l}\text { Explícito y replicable/con } \\
\text { tendencia al uso de GRADE }\end{array}$ & $\begin{array}{l}\text { Explícito y replicable/con } \\
\text { tendencia al uso de GRADE }\end{array}$ \\
\hline $\begin{array}{l}\text { Uso de recursos y costo de } \\
\text { elaboración }\end{array}$ & Bajo & Alto & Mediano \\
\hline
\end{tabular}

Elaboración propia. PICO: Población, Intervención, Comparador y Outcome; MBE: Medicina Basada en Evidencias; GRADE, Grading of Recommendations Assessment, Development and Evaluation.

los resultados o estudios con resultados heterogéneos, ausencia de evidencia directa en el grupo de interés e imprecisión de los resultados ${ }^{[3,35]}$.

En segundo lugar, porque las recomendaciones implican juicios de valor y estos pueden estar influenciadas por las opiniones, experiencia y la composición del grupo elaborador con el riesgo de que estas sean inferiores a otras opciones existentes o incluso perjudiciales.

En tercer lugar, debido a que en el desarrollo de las guías existen otras perspectivas además de las del paciente en su elaboración, como las del financiador, que pueden generar recomendaciones subóptimas con el fin de controlar los costos, atender a las necesidades de la sociedad o incluso de otros grupos de interés ${ }^{[3]}$. Estandarizar las intervenciones mediante GPC con recomendaciones generales en vez de plantear diferentes opciones de intervención podría ignorar la preferencia de los pacientes. Estas limitaciones a su vez pueden perturbar una buena relación médico-paciente ${ }^{[3,36]}$.
Para los médicos, las GPC defectuosas pueden comprometer la calidad de las atenciones que estos brindan y generar observaciones o incluso penalidades infundadas a sus prácticas. La existencia de guías con recomendaciones opuestas de diferentes grupos elaboradores puede confundir a los médicos y prolongar el uso de prácticas anticuadas y obsoletas. Los algoritmos propuestos con secuencia de decisiones binarias (sí/no) a menudo no reflejan la complejidad del acto médico y los procesos de pensamiento paralelos e iterativos inherentes al juicio clínico. También pueden desalentar el desarrollo de investigaciones cuando se concluye inadecuadamente que ciertas intervenciones son ineficaces ${ }^{[3]}$.

Los sistemas de salud y los financiadores pueden verse perjudicados por GPC cuando se compromete la eficiencia o desperdicia recursos limitados, principalmente cuando se recomiendan intervenciones costosas que son inasequibles. También pueden generar una disminución o retiro de las coberturas de ciertas intervenciones, o puede darse el caso en el que intervenciones costosas pueden desplazar los recursos 


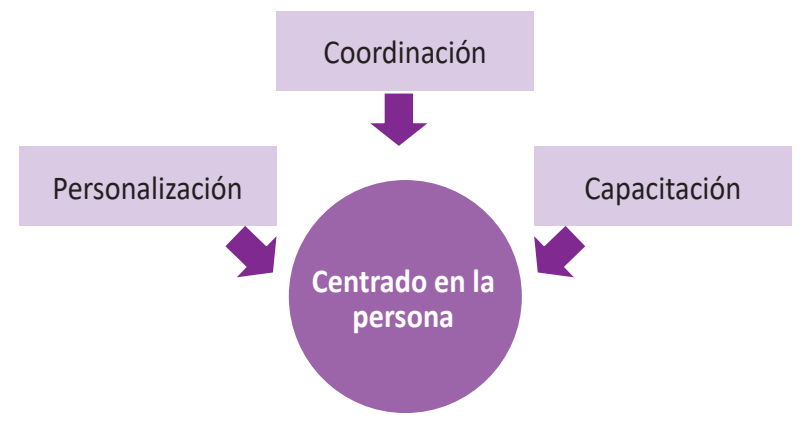

Dignidad, respeto, compasión

Figura 4. Cuidado centrado en la persona

siempre limitados desde intervenciones de enfermedades de mayor prevalencia o carga de una población ${ }^{[3]}$.

\section{GPC CON ENFOQUE CENTRADO EN LA PERSONA}

Una de las principales críticas que se realiza a las GPC es que al estandarizar los procesos de atención del paciente general descuida la individualización de la atención ${ }^{[3]}$. Sin embargo, importantes organismos desarrolladores como NICE han incorporado el enfoque de cuidado centrado en la persona, el que se fundamenta en cuatro principios: 1) Toda persona debe ser tratada con dignidad, respeto y compasión; 2) ofrecer cuidados o tratamientos coordinados; 3 ) ofrecer cuidados o tratamientos personalizados y; 4) estar capacitado para hacerlo ${ }^{[37,38]}$. (Figura 4).

\section{CONCLUSIONES}

- El desarrollo y la mejora de la calidad de las GPC ha sido progresivo y se ha dado gracias a la incorporación de metodologías claras, capacidad de los recursos humanos en su elaboración y preocupación institucional por alcanzar los mejores estándares.

- Las estrategias de elaboración y uso de GPC-BE están en constante evolución y su desarrollo se ve influenciado por factores de organización social de los sistemas de salud tanto en países desarrollados como en países en vías de desarrollo.

- Los países deben asegurar la calidad en el desarrollo de las GPC bajo un enfoque basado en evidencia y centrado en la persona que permita garantizar una mejor atención a los pacientes o usuarios de servicios de salud.

Fuente de financiamiento: El autor declara no haber recibido ninguna financiación para la realización de este trabajo.

Declaración de conflicto de intereses: Los autores declaran no tener conflicto de intereses con la publicación de este artículo.

\section{REFERENCIAS BIBLIOGRÁFICAS}

1. Chassin MR, Brook RH, Park RE, Keesey J, Fink A, Kosecoff J, et al. Variations in the use of medical and surgical services by the medicare population. N Engl J Med. 1986;314(5):285-90.

2. Welch WP, Miller ME, Welch HG, Fisher ES, Wennberg JE. Geographic variation in expenditures for physicians' services in the United States. N Engl J Med. 1993;328(9):621-7.

3. Woolf SH, Grol R, Hutchinson A, Eccles M, Grimshaw J. Clinical guidelines: potential benefits, limitations, and harms of clinical guidelines. BMJ. 1999;318(7182):527-30.

4. Paschke R, Hegedus L, Alexander E, Valcavi R, Papini E, Gharib H. Thyroid nodule guidelines: agreement, disagreement and need for future research. Nat Rev Endocrinol. 2011;7(6):354-61.

5. Sobieraj-Teague M, Eikelboom JW, Hirsh J. How can we reduce disagreement among guidelines for venous thromboembolism prevention? J Thromb Haemost. 2010;8(4):675-7.

6. Cruse H, Winiarek M, Marshburn J, Clark O, Djulbegovic B. Quality and methods of developing practice guidelines. BMC Health Serv Res. 2002;2(1):1.

7. Attia A. Adaptation of international evidence based clinical practice guidelines: The ADAPTE process. Middle East Fertil Soc J. 2013;18(2):123-6.

8. Grilli R, Magrini N, Penna A, Mura G, Liberati A. Practice guidelines developed by specialty societies: the need for a critical appraisal. Lancet. 2000;355(9198):103-6.

9. Alonso-Coello P, Irfan A, Sola I, Gich I, Delgado-Noguera M, Rigau $D$, et al. The quality of clinical practice guidelines over the last two decades: a systematic review of guideline appraisal studies. Qual Saf Health Care. 2010;19(6):e58.

10. Oxman AD, Lavis $J N$, Fretheim A. Use of evidence in WHO recommendations. World Hosp Health Serv. 2007;43(2):14-20.

11. Hill S, Pang T. Leading by example: a culture change at WHO. Lancet. 2007;369(9576):1842-4.

12. Sinclair D, Isba R, Kredo T, Zani B, Smith H, Garner P. World Health Organization guideline development: an evaluation. PloS One. 2013;8(5):e63715

13. Graham R, Mancher M, Miller Wolman D, Greenfield S, Steinberg $E$, editors. Clinical practice guidelines we can trust. Washington, DC: National Academies Press; 2011.

14. Reveiz L, Tellez DR, Castillo JS, Mosquera PA, Torres M, Cuervo LG, et al. Prioritization strategies in clinical practice guidelines development: a pilot study. Health Res Policy Syst. 2010;8:7.

15. Shekelle PG, Woolf SH, Eccles M, Grimshaw J. Developing guidelines. BMJ. 1999;318(7183):593-6.

16. Cook DJ, Greengold NL, Ellrodt AG, Weingarten SR. The relation between systematic reviews and practice guidelines. Ann Intern Med. 1997;127(3):210-6.

17. U.S. Department of Health and Human Services. National Guideline Clearinghouse [Internet]. Rockville, Maryland: Agency for Healthcare Research and Quality; 2016 [citado el 2 de abril de 2016]. Disponible en: https://www.guideline.gov

18. Guyatt GH, Oxman AD, Kunz R, Vist GE, Falck-Ytter Y, Schunemann HJ. What is "quality of evidence" and why is it important to clinicians? BMJ. 2008;336(7651):995-8.

19. Alonso-Coello P, Schunemann HJ, Moberg J, Brignardello-Petersen R, Akl EA, Davoli M, et al. GRADE Evidence to Decision (EtD) frameworks: a systematic and transparent approach to making well informed healthcare choices. 1: Introduction. BMJ. 2016;353:i2016. 
20. Guyatt GH, Oxman AD, Vist GE, Kunz R, Falck-Ytter Y, Alonso-Coello P, et al. GRADE: an emerging consensus on rating quality of evidence and strength of recommendations. BMJ. 2008;336(7650):924-6.

21. Development and validation of an international appraisal instrument for assessing the quality of clinical practice guidelines: the AGREE project. Qual Saf Health Care. 2003;12(1):18-23.

22. Makarski J, Brouwers MC; AGREE Enterprise. The AGREE Enterprise: a decade of advancing clinical practice guidelines. Implement Sci. 2014;9:103.

23. Brouwers M, Browman G, Burgers J, Cluzeau F, Davis D, Feder G. Instrumento AGREE II [Internet]. Consorcio AGREE; 2009 [citado el 2 de abril de 2016]. Disponible en: http://www.agreetrust.org/ wp-content/uploads/2013/06/AGREE II_Spanish.pdf

24. Darzi A, Abou-Jaoude EA, Agarwal A, Lakis C, Wiercioch W, Santesso N, et al. Frameworks for adaptation of health guidelines: a methodological survey. J Clin Epidemiol. 2017;86:3-10.

25. Bahtsevani C, Uden G, Willman A. Outcomes of evidence-based clinical practice guidelines: a systematic review. Int J Technol Assess Health Care. 2004;20(4):427-33.

26. Allen LA, O'Donnell CJ, Giugliano RP, Camargo CA, Jr., Lloyd-Jones DM. Care concordant with guidelines predicts decreased long-term mortality in patients with unstable angina pectoris and non-STelevation myocardial infarction. Am J Cardiol. 2004;93(10):1218-22.

27. Frei CR, Attridge RT, Mortensen EM, Restrepo MI, Yu Y, Oramasionwu $\mathrm{CU}$, et al. Guideline-concordant antibiotic use and survival among patients with community-acquired pneumonia admitted to the intensive care unit. Clin Ther. 2010;32(2):293-9.

28. Newton CA, Young S. Financial implications of glycemic control: results of an inpatient diabetes management program. Endocr Pract. 2006;12 Suppl 3:43-8.

29. Willis ZI, Duggan EM, Bucher BT, Pietsch JB, Milovancev M, Wharton W, et al. Effect of a clinical practice guideline for pediatric complicated appendicitis. JAMA Surg. 2016:e160194.
30. Shapiro DW, Lasker RD, Bindman AB, Lee PR. Containing costs while improving quality of care: the role of profiling and practice guidelines. Annu Rev Public Health. 1993;14:219-41.

31. Diercks DB, Roe MT, Chen AY, Peacock WF, Kirk JD, Pollack CV, Jr., et al. Prolonged emergency department stays of non-ST-segmentelevation myocardial infarction patients are associated with worse adherence to the American College of Cardiology/American Heart Association guidelines for management and increased adverse events. Ann Emerg Med. 2007;50(5):489-96.

32. Knaus SJ, Saum L, Cochard E, Prichard W, Skinner B, Medas R. Impact of evidence-based guidelines on outcomes of hospitalized patients with clostridium difficile infection. South Med J. 2016;109(3):14450 .

33. Beniwal LA, Kleven KL, Moody LT, Molin BM, Kantola SJ, Carlson $\mathrm{ML}$, et al. Outcomes of a quality improvement initiative to prevent unnecessary packed red blood cell transfusions among extremely low birth-weight neonates. Adv Neonatal Care. 2016;16(1):E3-9.

34. Kosimbei G, Hanson K, English M. Do clinical guidelines reduce clinician dependent costs? Health Res Policy Syst. 2011;9:24

35. Sanabria AJ, Rigau D, Rotaeche R, Selva A, Marzo-Castillejo M, Alonso-Coello P. [GRADE: Methodology for formulating and grading recommendations in clinical practice]. Aten Primaria. 2015;47(1):48-55

36. Guthrie B, Payne K, Alderson P, McMurdo MET, Mercer SW. Adapting clinical guidelines to take account of multimorbidity. BMJ. 2012; 345:e6341.

37. Collins A. Measuring what really matters [Internet]. London: Health Foundation; 2014 [citado el 16 de diciembre de 2016]. Disponible en: http://www.health.org.uk

38. Person-centred care made simple [Internet]. London: Health Foundation; 2014 [citado el 16 de diciembre de 2016]. Disponible en: http://www.health.org.uk

\section{Las ediciones anteriores de Acta Médica Peruana están disponibles en:}

www.scielo.org.pe

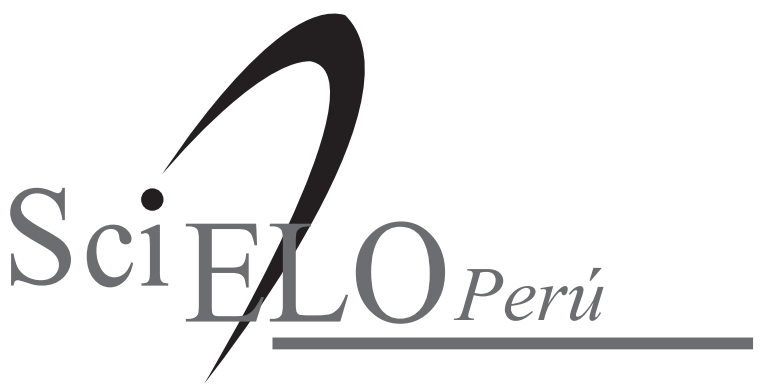

